\title{
3D Vector Topology Model in the Visualization System
}

\author{
Yan Xia Anthony Tung Shuen Ho \\ School of Electrical and Electronic Engineering \\ Nanyang Technological University, Singapore 639798 \\ Tel. (65)7905465, Fax: (65)7904161, E-mail address: eyxia@ntu.edu.sg
}

\begin{abstract}
To store, access, process and show the spatial geoinformational data easily in the microcomputer environment, the $3 \mathrm{D}$ model must be determined. The paper introduces $3 \mathrm{D}$ vector topology model in the Visualization System; first, to confirm entity characters in the System based on the information characters of three-dimensional entity in the world. Second, to study and define the $3 \mathrm{D}$ entity using the object-oriented approach, and to draw the conclusion -- there is only polyhedron geometric class in the $3 \mathrm{D}$ vector topological model. Furthermore, it is discussed how to build 3D vector topology model in the Visualization System. Third, to present what constitute a polyhedron, which relationships exist among the geometric elements, and how to represent the $3 \mathrm{D}$ geometric elements in the Visualization system using an example.
\end{abstract}

Keywords: 3D Vector Topology Model, Data Structure, Object-oriented, Topology Relationship

\section{INTRODUCTION}

$3 \mathrm{D}$ geological model is core to geological information visualization. Not the model fits to express the geometric shape and thematic characters of geological entity efficiently, but also the data structure describing the geometric shape and thematic character of $3 \mathrm{D}$ entity used in the model can be operated in the computer easily. Therefore, rational and efficient geoinformation model is the precondition of processing and showing of geoinformation[1].

$3 \mathrm{D}$ vector model is an only model describing $3 \mathrm{D}$ entity in the microcomputer environment. Many commissions, learned persons and wise men broke their heads and hurt their brains for many years on this problem of definitions of $3 \mathrm{D}$ entities or features and their thematic and geometric descriptions. Molenaar[2], Dr. Li[3] and others proposed the conceptual model of $3 \mathrm{D}$ vector GIS and failed to implement it practicably. The paper introduces $3 \mathrm{D}$ vector topology model in the Visualization System; first, to confirm entity characters in the System based on the information characters of threedimensional entity in the world. Second, to study and define the $3 \mathrm{D}$ entity using the object-oriented approach, and to draw the conclusion--there is only polyhedron geometric type in the 3D vector topological model. Furthermore, it is discussed how to build $3 \mathrm{D}$ vector topology model in the Visualization System. Third, to present what constitute a polyhedron, which relationships exist among the geometric elements, and how to represent the $3 \mathrm{D}$ geometric elements in the Visualization system using an example.

\section{INFORMATION·CHARACTERS OF 3D GEOLOGICAL ENTITY}

The district geological entity is composed of a series of sub-geological entities, which have different attributes (Fig 1). Each $3 \mathrm{D}$ geological entity has four information characters in the nature: thematic character, spatial position character, spatial relational character and time character.

- Thematic Character: describing the phenomenal or character of geological entity, i.e. the age of geological entity, the thematic character of geological entity. It is displayed by the graphics symbol or the color in the graphics, is represented by a group of numbers or characters in the computer.

- Spatial Position Character: the entity is displayed abstractly by one of 3D geometric objects -- point, line and face or polyhedron (body), in the graphics; it is described by a serial of $(x, y, z)$ coordinates in the computer according to the graphics characters of point, line, face or body.

- Spatial Relation Character: describing the relation of spatial neighboring and containing among sub-entities. The spatial relation of sub-entity is identified by the difference of graphics or colors in the graphics, and defined by the topological structure among the spatial data in the computer.

- Time Character: the changing character of entity according to time.

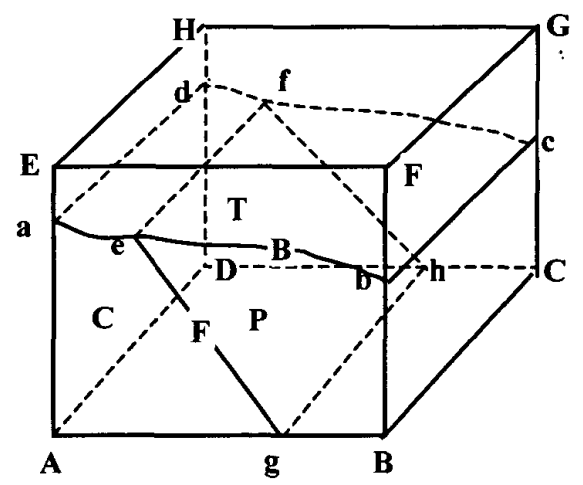

Figure 1 Region Geological Entity 


\section{OBJECT-ORIENTED DESCRIPTION OF 3D GEOLOGICAL ENTITY}

3D geological entity is a collection of a series of thematic (physical, chimerical) and relations in the geological domain. The steps of object-oriented description of 3D geological entity are first, to determine the class and thematic character of geological entity based on the aim of research and application. After the thematic characters of different entities are determined, only the thematic characters closing with the aim of research and application are kept, otherwise must be discarded, so that geological object has a closely connection with a series of characters, and the thematic and spatial analysis can be finished easily. For example, in order to analyze the region geological structure, we divide the region geological entity into C, P, T, F and B sub-geological entities based on the age and thematic characters, Fig. 1. Second, to define 3D spatial geometric model of the object. Based on the 3D spatial geometric shape, the 3D object can be divided into point, line, face and body four different geometric object classes. Each object class is consisted of lots of points, lines, and faces geometric elements (Fig. 1). The spatial position character of the entity can be determined by the $(x, y, z)$ coordinates of the geometric elements. The spatial relational characters among the entities are described by the topological characters among the geometric elements of the geometric class objects.

Consequently, the following steps must be done in order to build $3 \mathrm{D}$ vector topological model: first, to determine the class and thematic character of geological entity based on the aim of research and application. Second, to define 3D geometric characters of the entities. That is, defining the geometric classes of the geological entity, confirming which points, lines, and faces consist of the geometric class, and the $3 \mathrm{D}$ topological relations among the geometric elements.

\section{D VECTOR TOPOLOGYCAL GEOLOGICAL MODEL}

\section{Polyhedron Object}

Supposed researching the region geological structure, we divide the region geological entity into $\mathrm{C}, \mathrm{P}, \mathrm{T}, \mathrm{F}$ and $\mathrm{B}$ subgeological entities based on the age and thematic characters, Fig.1. Each sub-entity is a different thematic object in the $3 \mathrm{D}$, and defined by one or more enclosing directional surfaces, the coordinates in the surfaces determins the shape and position of each sub-entity.

\section{Geometrical Character of Polyhedron Entity}

In the conceptual model of $3 \mathrm{D}$ vector GIS, 3D entity in the nature is regarded as one of the point, line, face and body four basic spatial object classes abstractly $[2,3]$. But in the $3 \mathrm{D}$ vector topological model, there is only polyhedron class; each polyhedron entity has both thematic and geometric characters. Point object is abstractly belonged to polyhedron class, its volume and out-surface area equal to zero. The point object enclosed in out-surface and having the thematic of enclosed thematic can be discarded. Line object cannot be existed in the geological domain. Face object, i.e. fault, disconnection, also is regarded as the body object having the characters of zero-volume, some zero-area faces and some zero-length lines. So, point, line, face and body four basic spatial objects are abstractly regarded as polyhedron geometrical object in the paper. That is, there is only one geometrical object class in the $3 \mathrm{D}$ vector topological model -- polyhedron (body) geometrical class [4].

\section{Geometrical Element of Polyhedron Entity}

All of $3 \mathrm{D}$ entities in the geoinformation visualization system must be data that can be stored in the computer. In Fig. 1, the region geological entity is enclosed by six outsections, one or more directional enclosed faces define the spatial boundary of each sub-entities. Each out-section is composed of lots of faces having different characters, and its boundary is defined by a lot of arris. For each surface, the boundary is determined by one or more directional enclosed curves, the form and position are defined by the pointcoordinates lying on the surface and the boundary, the thematic is defined by the point's thematic. The boundary of the face (surface, out-section, inter-section) is defined by an out-circle. The circle is an enclosed boundary which is composed by a series of sequence and directional edges or arris, the out-circle defines the maximum boundary of face. The form of face is controlled by control-points and can be represented by arcs or circles. The neighboring surfaces of polyhedron object (included sub-body) cross on the edge or arris, the surface of body contains lots of arcs. A starting node and an ending node controls the direction of these edges and arris, the form of edge or arris is controlled by lots of control points and defined by lots of interpolation points.

Therefore, the region entity is divided into series subentities according to the research's aim. Each sub-entity is enclosed by lots of surfaces, and its spatial shape is polyhedron. Each face's boundary is defined by lots of edges or arris, each edge or arris contains at least two nodes. Each node connects more than two edges or arris. The relationship between body object and the geometric elements of point, line and face is a multi-hierarchic network in the $3 \mathrm{D}$ vector topological model.

\section{Topological Relationship Among Geometrical Elements}

A polyhedron object is defined by lots of point, line, and face geometrical elements. And, there are two important information among the geometrical elements of polyhedron: one is geometrical information, it depicts the character and measurement of geometrical elements, i.e. position, size. Another is connective relation among geometrical elements, which is topological information. It represents that which faces define the polyhedron, which circle encloses the face and is composed of which edges and arris, and which nodes defines the edge or arris, and so on. Concerning multi-factors, 
seven topological relations of geometrical element in the Visualization system are confirmed. (Table 1, Table 2 Table 3, Table 4, Table 5, Table 6, and Table 7).

Table 1 Polyhedron Topological Relation

\begin{tabular}{|l|l|l|}
\hline Body & \multicolumn{1}{|c|}{ Surface } & \multicolumn{1}{|c|}{ Neighbor Body } \\
\hline-1 & ABFE,DHGC,DAEH,BCGF,EFGH,BADC & $-1,-1,-1,-1,-1,-1$ \\
$\mathrm{~T}$ & bFEa, cdHG, aEHd, GFbc, EFGH, badc & $-1,-1,-1,-1,-1, \mathrm{~B}$ \\
$\mathrm{P}$ & begB, cChf, efhg, cbCB, ebcf, ghCB & $-1,-1, \mathrm{~F},-1, \mathrm{~B},-1$ \\
$\mathrm{C}$ & eaAg, fhDd,adDA, fegh, daef, ADhg & $-1,-1,-1, \mathrm{~F}, \mathrm{~B},-1$ \\
$\mathrm{~B}$ & baab, decd, adda, cbbc, effe, daef,ebcf & $-1,-1,-1,-1, \mathrm{~T}, \mathrm{C}, \mathrm{F}, \mathrm{P}$ \\
$\mathrm{F}$ & geeg,fhhf,efhg,fegh,feef,ghhg & $-1,-1, \mathrm{C}, \mathrm{P}, \mathrm{B},-1$ \\
\hline
\end{tabular}

Table 2 Section Topological Relation

\begin{tabular}{|c|c|c|c|c|c|}
\hline Section & Outcircle & \multicolumn{1}{|c|}{ Faces } & Arris and Edge & PosiBody & NegBody \\
\hline ABFE & ABFE & $\begin{array}{l}\text { eaAg,begB,geeg } \\
\text { baab, bFEa }\end{array}$ & B,BF,-EF-AE & -1 & C,P,F,B,T \\
DHGC & DHGC & $\begin{array}{l}\text { cdHG, cChf, } \\
\text { fhDd, dccd, fhhf } \\
\text { aEHd, adda, } \\
\text { DAEH }\end{array}$ & H,HG,-CG,-DC & -1 & AD,A, A, C,B,FH,-DH \\
$\ldots$ & $\ldots$ & -1 & T,B,C \\
\end{tabular}

Table 3 Surface Topological Relation

\begin{tabular}{|c|c|c|c|}
\hline Surface & Out-circle & Posi Neighboring Body & Neg Neighboring Body \\
\hline bFEa & bFEa & -1 & $\mathrm{~T}$ \\
cdHG & cdHG & -1 & $\mathrm{~T}$ \\
aEHd & aEHd & -1 & $\mathrm{~T}$ \\
$\ldots$ & $\ldots$ & $\ldots$ & $\ldots$ \\
\hline
\end{tabular}

Table 4 Circle Topological Relation

\begin{tabular}{|c|l|l|}
\hline \multicolumn{1}{|c|}{ Circle } & \multicolumn{1}{|c|}{ Edge,Arris } & \multicolumn{1}{c|}{ Internal Surface } \\
\hline $\mathrm{bFEa}$ & $\mathrm{bF},-\mathrm{EF}, \mathrm{-aE}, \mathrm{ab}$ & $\mathrm{bFEa}$ \\
$\mathrm{cdHG}$ & $-\mathrm{dc}, \mathrm{dH}, \mathrm{HG},-\mathrm{cG}$ & $\mathrm{cdHG}$ \\
$\mathrm{AEHd}$ & $\mathrm{aE}, \mathrm{EH}, \mathrm{dH},-\mathrm{da}$ & $\mathrm{aEHd}$ \\
$\ldots$ & $\ldots$ & \\
\hline
\end{tabular}

Table 5 Arris Topological Relation

\begin{tabular}{|l|l|l|l|c|}
\hline \multicolumn{1}{|c|}{ Arris } & Starting Node & Ending Node & Edges, Arris & Out-sections \\
\hline $\mathrm{AB}$ & $\mathrm{A}$ & $\mathrm{B}$ & Ag,gg,gB & ABFE,-BADC \\
$\mathrm{BC}$ & $\mathrm{B}$ & $\mathrm{C}$ & $\mathrm{BC}$ & CGFB,-BADC \\
$\mathrm{DC}$ & $\mathrm{D}$ & $\mathrm{C}$ & Dh,hh,hC & BADC,-GCDH \\
$\mathrm{AD}$ & $\mathrm{A}$ & $\mathrm{D}$ & $\mathrm{AD}$ & $\mathrm{BADC}, \mathrm{HDAE}$ \\
$\ldots$ & & & $\ldots$ & $\ldots$ \\
\hline
\end{tabular}

Table 6 Edge and Arris Topological Relation

\begin{tabular}{|c|c|c|c|}
\hline Edge, Arris & Starting Node & Ending Node & Circle \\
\hline $\mathrm{bF}$ & $\mathrm{b}$ & $\mathrm{F}$ & bFEa, GFbc \\
$\mathrm{Bb}$ & $\mathrm{B}$ & $\mathrm{b}$ & Bbeg, bBCc \\
$\ldots$ & & & \\
\hline
\end{tabular}

Table 7 Node Topological Relation

\begin{tabular}{|c|c|c|}
\hline Node & Starting Edge $\square$ Arris & Ending Edge $\square$ Arris \\
\hline $\mathrm{b}$ & $\mathrm{bF}, \mathrm{be}$ & $\mathrm{cb}, \mathrm{Bb}$ \\
$\mathrm{B}$ & $\mathrm{Bb}, \mathrm{Bg}$ & $\mathrm{CB}$ \\
$\ldots$ & & \\
\hline
\end{tabular}

\section{CONCLUSION}

- 3D geological entity has four information characters: thematic character, spatial position character, spatial relational character and time character.

- In order to build 3D vector structure model, the needed works are: first, to determine the classification and the thematic character of the geological entity. Second, to determine the geometric characters of the geological entity, i.e. the geometric class of the entity, the geometric class consists of which points, lines, and faces, and the $3 \mathrm{D}$ topological relations among the geometric elements.

- Concerning the geological domain, the point, line, face, and body spatial geometric objects are abstractly regarded as body objects. There is only one geometric class--polyhedron in the 3D Vector structure model.

- 3D region entity is composed of a series of sub-entities, each has both of thematic and geometric characters. Each sub-entity is enclosed by a lot of faces. The boundary of each face is composed of some edges and arris. Each edge and arris contains two nodes. Each node connects more than two edges and arris. The relationship between sub-entity and point, line, face is a multi-hierarchic network.

\section{REFERENCE}

[1] Martien Molenaar, Object Hierarchies and Uncertainty in GIS or why is Standardization so Difficult? 1993, GIS,4 Vol.6

[2] Martien Molenaar, A Topology for 3D Vector Maps, ITC Journal 1992-1

[3]Qingyuan Li, Study on the topological relation of 3D Vector GIS, Paper of Ph.D of China University of Mining and Technology, 1996

[4] Yan Xia, Study on 3D Vector Topology Geological Model and Geoinformatics Visualization System, Paper of Ph.D of China University of Mining and Technology, 1997 